\title{
SEMICONDUCTOR OPTICAL AMPLIFIERS IN AVIONICS
}

C Michie (1), W Johnstone (1), I Andonovic (1), E Murphy (2), H White (2) A Kelly (3)

(1) University of Strathclyde, Glasgow, UK

(2) BAE Systems Advanced Technology Centre, Bristol, UK

(3) University of Glasgow, Glasgow, UK

\section{Introduction}

It has long been recognised that significant benefits, e.g. immunity to electromagnetic interference (EMI), weight savings, data rate scalability, are obtainable using optical fibre communications within avionics environment. Harnessing the advances made in commercial communications technologies and standards offers a cost effective route to realising that potential, provided that the harsh operational demands e.g. temperature range $-55^{\circ} \mathrm{C}$ to $+125^{\circ} \mathrm{C}$ can be met. Long haul communications links employ Dense Wavelength Division Multiplexing (DWDM), an approach that maximises the usage of the available optical fibre transmission bandwidth; tens of individual wavelengths, each carrying up to $40 \mathrm{Gbit} / \mathrm{s}$ data rate, are coupled into a single mode optical fibre. This requires a range of single wavelength sources, each wavelength stabilised to within a fraction of a nanometre in order to function. This stringent referencing of the source wavelength is not appropriate for avionic systems; however approaches to provide DWDM overlays within Passive Optical Networks (PONs) for the access layer of the network, may provide an appropriate solution. The progress towards implementing such a system, considering specifically the issues of minimising source inventory, cost and optimising operating temperature range using minimal cooling to make the system function is reported.

\section{DWDM PON Architectures For Avionics}

Reflective Semiconductor Optical Amplifiers (RSOA) are a promising device that underpins the delivery of DWDM capability cost effectively without the need for wavelength specific sources [1]. RSOAs can be directly modulated by changing their bias currents at data rates up to and beyond $1 \mathrm{Gbit} / \mathrm{s}$. They provide the core capabilities to simultaneously amplify signals while at the same time modulating them with a data stream. The concept is illustrated in Figure 1a. A broadband light source (BLS) is spectrum sliced using a wavelength demultiplexer. The transmitted light is then used to seed an RSOA which can be modulated to act as a transmitter. The power entering the RSOA is small but it is amplified as well as modulated and significant power levels are returned to the network. In principle an RSOA can be used as the BLS.

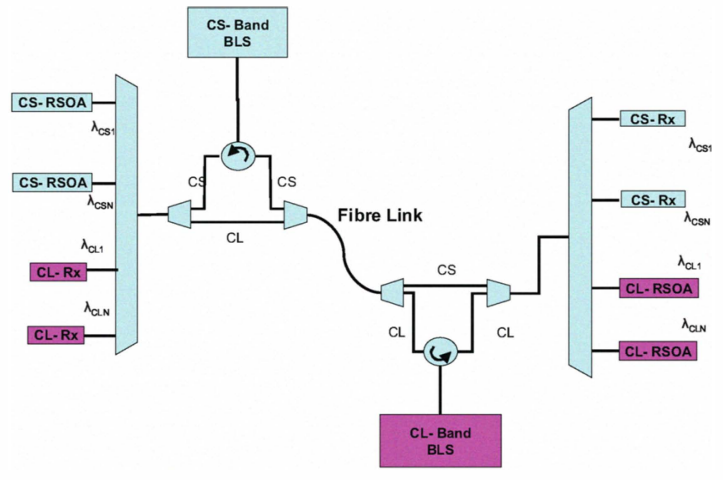

(a)

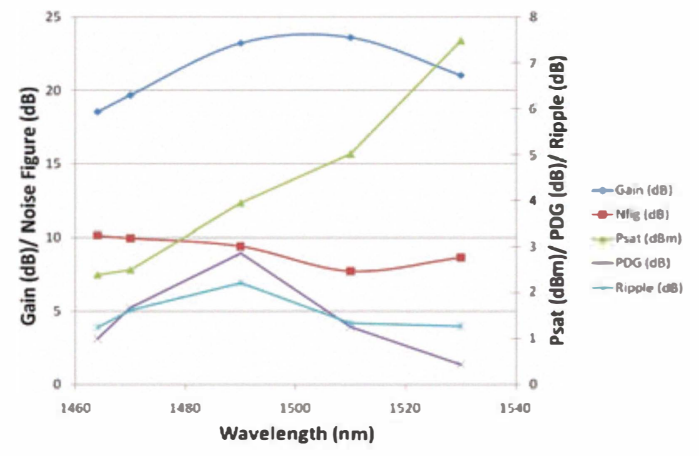

(b)

Figure 1: (a) Schematic of a white light based DWDM PON (b) Typical parametric performance of an RSOA

\section{Parametric Performance of RSOA and Path Loss Capability (PLC)}

The parametric performance of a commercial RSOA is displayed in Figure 1 (b) Over the wavelength range $1380 \mathrm{~nm}$ to $1550 \mathrm{~nm}$, the device provides more than $20 \mathrm{~dB}$ gain and a maximum output power $\left(\mathrm{P}_{\mathrm{SAT}}\right)$ of 3 
$\mathrm{dBm}$. Thus seeding the RSOA with a signal of $-17 \mathrm{dBm}$ or more translates into a signal power into the fibre of 3 $\mathrm{dBm}$. Similar performances are obtainable over the $\mathrm{C}$ and $\mathrm{L}$ band (up to $1600 \mathrm{~nm}$ ). The path loss capability (PLC), the loss that can be tolerated between the transmitter and the receiver in a network while maintaining acceptable Bit Error Rate e.g. $10^{-12}$, is a function of the power that can be delivered into the network from the transmitter and the receiver sensitivity. A typically PON enjoys a PLC of $20 \mathrm{~dB}$, representing a transmission loss of $20 \mathrm{~km}(5 \mathrm{~dB})$, a 16 way split $(12 \mathrm{~dB})$ and $3 \mathrm{~dB}$ for component ageing and connectors. Typical COTS devices offer an additional $3 \mathrm{~dB}$ to $5 \mathrm{~dB}$ of margin. In the absence of any defined standard for avionics, this was deemed an appropriate metric. Typical COTS receivers provide a minimum sensitivity of $<-22 \mathrm{dBm}$ for a BER of $10^{-12}$ at $2.5 \mathrm{Gbit} / \mathrm{s}$. Using an RSOA, a PLC of more than $20 \mathrm{~dB}$ is readily obtainable.

The performance of a wavelength seeded RSOA PONs using RSOAs to amplify the seed signal and directly modulate it with data to be carried in the upstream direction has been displayed in Figure 2. The transmission (backhaul and splitter) was represented by a lumped loss since at low data rates $(1.25 \mathrm{Gbit} / \mathrm{s})$ dispersion is not significant. The seed wavelength was injected into an RSOA, directly modulated at $1.25 \mathrm{Gbits} / \mathrm{s}$ with a NRZ Pseudo Random Bit Sequence (PRBS) of $2^{11}-1$. Initial characterisations were performed at a chip temperature of $25^{\circ} \mathrm{C}$. In this characterisation, the upstream modulated signal was detected at the Optical Line Terminal (OLT) using combination of an Avalanche Photodiode (New Focus 1647 APD Photo-receiver) followed by a limiting amplifier. This gives a better performance than would be expected from a PON since the receiver is more sensitive $\left(-29 \mathrm{dBm}\right.$ for BER $\left.10^{-9}\right)$ but nevertheless, the wavelength multiplexing evaluation is valid. The BER was evaluated by an Agilent N4903A J-BERT Bit Error Rate Analyser. The PLC of the RSOAs was assessed over a range of wavelengths covering the S,C and L bands using the two RSOAs at a seed power of $-20 \mathrm{dBm}$ (Figure 2), representative of a typical system condition. Each device is capable of providing $>25 \mathrm{~dB}$ PLC over a $60 \mathrm{~nm}$ span. The combination of the RSOAs provides a PLC of $>25 \mathrm{~dB}$ over a wavelength interval of $>130 \mathrm{~nm}$ (extending from $1460 \mathrm{~nm}$ to $1590 \mathrm{~nm}$ ).

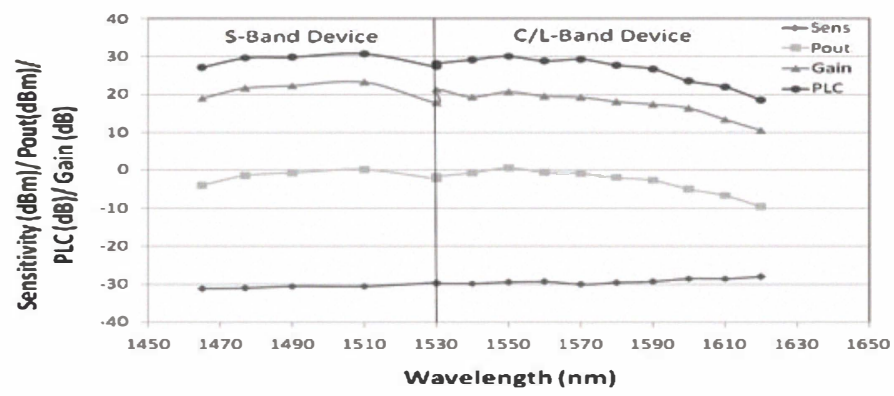

Figure 2: System characterisation as a function of wavelength.

\section{CONCLUSIONS}

RSOAs have been demonstrated to operate within a WDM PON architectures over a $>60 \mathrm{~nm}$ wavelength range with large path loss capabilities. Two RSOAs enable contiguous operation over the $\mathrm{S}, \mathrm{C}$ and $\mathrm{L}$ bands; results indicate clearly that WDM architectures suitable for avionics with a PLC of $>25 \mathrm{~dB}$ are possible with only two devices. Performance at extended temperature ranges will be reported later in detail.

\section{REFERENCES}

[1] R.P. Davey , D.B. Payne, 'The future of optical transmission in access and metro networks; an operator's view', ECOC, Vol. 5, pp53-56, 2005

[2] A Kelly, C Michie, Wende Zhong, S Karagianopoulos, W I Madden, C Tombling, I Andonovic, 'High Performance Polarisation Independent Reflective Semiconductor Optical Amplifiers in the S, C and L bands',. IEEE Journal on Selected Areas in Communications, 28(6) 2010 DOI 10.1109/JSAC.2010.100818 